\title{
Bidding and managing congestion across multiple electricity spot markets
}

\author{
Adamantios Marinakis $\quad$ Anastasios G. Bakirtzis, Senior Member, IEEE Thierry Van Cutsem, Fellow, IEEE
}

\begin{abstract}
The possibility for market participants to place their bids in markets where they are not geographically located is investigated in this paper. A framework for managing the resulting congestion is proposed. The procedure is illustrated and commented on a test system.
\end{abstract}

Keywords. electricity spot markets, congestion management, market coupling, overlapping markets

\section{INTRODUCTION}

The creation of the Internal Electricity Market in Europe has been subject to lots of discussions in the last years, led basically by ETSO (European Transmission System Operators) and EuroPEX (European Power Exchanges) [1]. It seems that the prevailing mechanism is presently the market coupling method, where a coordinated market takes place among different markets, using each area's own rules [2]. First each sub-market is cleared and then these markets are coupled. The method is already in use since 2006, coupling the day-ahead markets of France, Belgium and the Netherlands [3], while it is to be enlarged to Germany and Luxembourg in 2009 [4]. These initiatives apply for the moment only in day-ahead markets. Steps are also taken towards opening intra-day and real-time markets to foreign participants [5].

In this paper, another way for coupling markets and handling inter-area congestion is investigated. The approach consists in allowing participants (generators, large consumers) to bid directly in any market of the interconnection they wish, without being obliged to do so via their local operator. Depending on the time frame, the term "operator" may correspond to a different entity. In day-ahead it is typically the Power Exchange (PX) while in real-time it is the Transmission System Operator (TSO). The term Market Operator (MO) is used in the sequel without distinction. So, the idea is that every market participant may choose the MO in whose market he wishes to participate.

Were it not for the transmission network constraints, the above would cause no trouble; every MO would just have to dispatch the actors who placed their bids in its market. Obviously these constraints need some treatment and it will definitely not be enough to have every MO responsible for an area of the interconnection since this MO may be dispatching market participants located in another geographical area. Actually, the assumption made in this paper (market participants

A. Marinakis (marinakis@montefiore.ulg.ac.be) is Ph.D. student at the Dept. of Electrical Engineering and Computer Science (Montefiore Institute) of the University of Liège, Sart Tilman B37, B-4000 Liège, Belgium.

T. Van Cutsem (t.vancutsem@ulg.ac.be) is a research director of FNRS (Fund for Scientific Research) and adjunct professor at the same department.

A.G. Bakirtzis (bakiana@eng.auth.gr) is professor at the Dept. of Electrical and Computer Engineering of the Aristotle University of Thessaloniki, Greece. bidding outside their geographical area) results in the appearance of overlapping markets which should be simultaneously cleared ensuring at the same time the feasibility of the final schedule.

In [6], precisely, the author investigates the modeling of this situation and proposes two iterative algorithms to perform the clearing sought. The global congestion management problem is decomposed into a "master problem" (coordination problem) and several "subproblems" (market problems). In the first proposed approach, the master problem determines congestion path prices and the subproblems solve for the individual market schedules and their own prices. In the second approach, instead of setting prices for the congested paths, a transmission coordinator simply allocates the total MW capacities of the paths to all markets in the master problem. Using allocated values as the path limits, the market operators solve for the subproblems.

The approach considered in this paper is similar to the second one outlined above in the sense that a central coordinator is in charge of allocating congested transmission capacity to the MOs. However, the problem is not treated as a global market clearing; instead, each market clearing is undisclosed to other markets. Thus, a different transmission sharing approach results.

The paper is organized as follows. In Section II the situation described in the Introduction is mathematically formulated. In Section III the proposed algorithm is presented, while in Section IV it is applied to a test case. The steps of the algorithm are explained in some detail and the results are evaluated. Finally, in the Conclusion, the approach is summarized and a possible future extension is commented.

\section{PROBLEM FORMULATION}

To facilitate the presentation, each $\mathrm{MO}$ is assumed to represent the loads of a certain region and resort to generators all around the interconnection in order to satisfy its demand.

Let $\mathbf{g}^{m}$ be the vector of all generators placing a bid in the market of the $m$-th $\mathrm{MO}, \mathbf{c}^{m}$ the vector of their corresponding bids and $\overline{\mathrm{g}}^{m}$ their maximum available quantities. Let us also denote by $\mathbf{d}^{m}$ the vector of loads that should be served by the $m$-th MO. Each MO will try to minimize the social cost (i.e. maximize the social welfare) by solving the following optimization problem:

$$
\begin{gathered}
\min _{\mathbf{g}^{m}} \mathbf{c}^{m T} \mathbf{g}^{m} \\
\text { s. t. } \quad \sum_{i} g_{i}^{m}=\sum_{j} d_{j}^{m} \\
0 \leq g_{i}^{m} \leq \bar{g}_{i}^{m}
\end{gathered}
$$


As is typical in such problems, a DC model is used to represent the network. More precisely, a bus to bus Power Transfer Distribution Factor (PTDF) sensitivity matrix $\mathbf{S}$ is used [7]. It refers to the entire interconnection and links line active power flows to bus active power injections. That is, with $\mathrm{g}$ the vector of total bus generations and $\mathbf{d}$ the one of total bus consumptions, the vector $\mathbf{p}$ of line power flows is given by:

$$
\mathbf{p}=\mathbf{S}(\mathbf{g}-\mathbf{d})
$$

where, in order to come up with the two-dimensional matrix $\mathbf{S}$, a slack bus has been considered and every bus-to-bus transaction has been expressed as a pair of two transactions, one towards and another from the slack bus. The choice of the slack bus does not affect the computed power flows.

Vectors $\mathbf{g}$ and $\mathbf{d}$ are computed by adding respectively the generations and loads allocated and served by all MOs, according to:

$$
\begin{aligned}
& \mathbf{g}=\sum_{m=1}^{N} \mathbf{g}^{m} \\
& \mathbf{d}=\sum_{m=1}^{N} \mathbf{d}^{m}
\end{aligned}
$$

where $N$ is the number of the MOs, each of them operating a market in the interconnection.

Let us call $\mathrm{g}^{m}$ the solution of problem (1) solved by the $m$-th MO. Assembling together all these solutions, and using (2)-(4), one easily obtains the resulting line flows $\mathbf{p}$. The case of interest here is when there are overloaded lines, i.e. $p_{l}>\bar{p}_{l}$ or $p_{l}<-\bar{p}_{l}$ for some $l$, where $\bar{p}_{l}$ the maximum capacity of line $l$.

\section{Presentation OF THE Algorithm}

\section{A. Overview}

This paper proposes an iterative algorithm to deal with the congestion management problem. Its principle consists in a gradual incorporation into the $\mathrm{MO}$ market clearing problem (1) of constraints on the resulting line power flows. That is, after the MOs have simultaneously and independently solved their optimization problems (1), the resulting schedules are assembled, the vector of bus power injections $\mathbf{g}-\mathbf{d}$ is constructed and the power flows are computed according to (2). Then a set of constraints is communicated to the MOs, for inclusion in their market clearing problems. These constraints are such that they unload the lines that have been found overloaded according to the previous bus injection schedules. The procedure is to be repeated until a feasible equilibrium is reached.

A central coordinator is assumed to be in operation, whose role is to compute the constraints sent to the MOs at every iteration. It is expected to be operated jointly by the involved TSOs and MOs (and maybe the market participants). In addition, TSOs are assumed to bring together the necessary information to come up with and share the DC model (2) of the entire interconnection. It is through this model that the central coordinator checks the feasibility of the MOs schedules.

\section{B. Sharing the congested transmission capacity}

The approach followed in the paper makes use of the linearity of the model (2) to decouple the line limits constraints and share them among the several MOs in a way that preserves independence of the various market clearings. Equations (2)(4) give:

$$
\mathbf{p}=\mathbf{S}\left(\sum_{m} \mathbf{g}^{m}-\sum_{m} \mathbf{d}^{m}\right)
$$

which is equivalent to

$$
\mathbf{p}=\sum_{m} \mathbf{p}^{m}
$$

with

$$
\mathbf{p}^{m}=\mathbf{S}\left(\mathbf{g}^{m}-\mathbf{d}^{m}\right)
$$

where $\mathbf{g}^{m}$ and $\mathbf{d}^{m}$ have dimensions equal to the total number of buses in the interconnection, with zero components at buses with no generation (respectively load) serving for (being served by) the $m$-th MO.

An interpretation of (7) is that $\mathbf{p}^{m}$ is the vector of line power flows caused by the schedule of market $m$. This decomposition of responsibility over the line flows is made possible by the use of the linear DC model.

Equation (7) suggests that the power flow constraint on the $l$-th line, originally expressed as

$$
-\bar{p}_{l} \leq p_{l} \leq \bar{p}_{l} \quad \Leftrightarrow \quad-\bar{p}_{l} \leq \mathbf{S}_{l}(\mathbf{g}-\mathbf{d}) \leq \bar{p}_{l}
$$

where $\mathbf{S}_{l}$ is the $l$-th row vector of matrix $\mathbf{S}$, can be decomposed into:

$$
\begin{gathered}
-\bar{p}_{l}^{m} \leq p_{l}^{m} \leq \bar{p}_{l}^{m} \\
\text { with } \quad \sum_{m} \bar{p}_{l}^{m}=\bar{p}_{l}
\end{gathered}
$$

So, it is suggested that for each line $l$ found overloaded, the central coordinator communicates to each $\mathrm{MO}$ a limit $\bar{p}_{l}^{m}$ on the power flow the MO creates in the congested line. In other words, the central coordinator allocates some line's transmission capacity to every MO.

Denoting by $\mathbf{p}_{L}$ the vector of line flows above limit after an iteration $k$ of the algorithm, the principle is that at the $k+1$ iteration, the $m$-th $\mathrm{MO}$ incorporates the following set of constraints into its market clearing problem (1):

$$
-\overline{\mathbf{p}}_{L}^{m} \leq \mathbf{p}_{L}^{m} \leq \overline{\mathbf{p}}_{L}^{m}
$$

The vectors $\overline{\mathbf{p}}_{L}^{m}$ are computed by the central coordinator and will reflect its congestion management policy. They are updated at each iteration $k$.

\section{Congestion management policy}

As is well known from optimization theory [8], if the coordinator seeks for minimizing the global social cost:

$$
S C=\sum_{m} \mathbf{c}^{m T} \mathbf{g}^{m}
$$

it should allocate the transmission line capacities in such a way that, for each congested line, the value of its use is equal for all the MOs. Indeed, the opposite, i.e. having at least one 
line $l$ for which the value $\lambda_{l}^{1}$ for MO-1 is larger than the value $\lambda_{l}^{2}$ for another MO-2 would suggest that $S C$ could be further decreased by de-allocating some capacity from MO-2 and allocating it to MO-1.

This is what has been done in [6], in which the author suggests that the MOs send to the central coordinator (referred to as "master problem" in [6]) the sensitivities of their cost functions to the line available capacities. Using this information, for all congested lines, the coordinator shares their available capacities among MOs so that they have the same value for everyone of them.

However, there might be no motivation for the MOs to compute and announce truly the values of the sensitivities under question. On the contrary, it would be in the interest of every MO to declare higher sensitivities since this will make it receive more transmission capacity. This was not an issue in [6], where the congestion management was treated as a decentralized optimization problem, with the single objective of minimizing the global social cost. On the contrary, the approach followed in this paper considers the involved MOs as competitors for the use of the transmission network (and in the longer term for the attraction of the cheapest generators). Their acknowledgement of the need of a coordinated operation as well as their will to participate into a coordination scheme are not questioned, but such a scheme should not be based on them providing voluntarily private information just for the sake of global optimization.

An alternative would be, at each iteration of the algorithm, to ask the MOs to announce their prices for the use of the congested lines. Then, the transmission capacity allocation could be made based on these prices. Of course, the MOs would have to pay for the use of the transmission network according to the prices they announced. This would be an explicit auctioning of the transmission capacity mechanism incorporated into the iterative coordination algorithm.

The approach proposed in this paper consists in considering that the larger the power flow a MO is causing in a transmission line $l$, the more this line is valuable to its schedule. Following this reasoning, the congestion management policy consists in sharing, at each iteration $k$, the transmission capacity of congested lines proportionally to their present use by the MOs. This gives the following limit for a congested line $l$, to be incorporated into the MOs market clearing at the next iteration:

$$
\bar{p}_{l}^{m(k+1)}=\frac{p_{l}^{m(k)}}{p_{l}^{(k)}} \bar{p}_{l}
$$

where $p_{l}^{(k)}$ is the power flow in line $l$ after the $k$-th iteration of the procedure and $p_{l}^{m(k)}$ the part of this flow attributed to the schedule of the $m$-th MO. It can be easily checked that this decomposition satisfies (9b).

The same approach can be regarded from another viewpoint as well; the more flow a MO is causing in an overloaded line, the more this MO should participate in the alleviation.

Indeed, with (12) satisfied, at the next iteration we have:

$$
p_{l}^{m(k+1)} \leq \frac{p_{l}^{m(k)}}{p_{l}^{(k)}} \bar{p}_{l}
$$

Calling $\delta_{l}^{m}$ the change in the power flow $p_{l}$ caused by the $m$-th MO, i.e. $\delta_{l}^{m}=p_{l}^{m(k+1)}-p_{l}^{m(k)}$, Eq. (13) becomes:

$$
\delta_{l}^{m} \leq \frac{p_{l}^{m(k)}}{p_{l}^{(k)}} \bar{p}_{l}-p_{l}^{m(k)}=\frac{p_{l}^{m(k)}}{p_{l}^{(k)}}\left(\bar{p}_{l}-p_{l}^{(k)}\right)
$$

The result is the same as if the MOs were asked to decrease their participation $p_{l}^{m}$ in the power flow by a minimum amount $\Delta p_{l}^{m}$ :

$$
\delta_{l}^{m} \leq \Delta p_{l}^{m}=\frac{p_{l}^{m(k)}}{p_{l}^{(k)}}\left(\bar{p}_{l}-p_{l}^{(k)}\right)
$$

thus satisfying (14).

In both viewpoints (use of constraint (13) or of (15)), the total line flow change is $\delta_{l}=p_{l}^{(k+1)}-p_{l}^{(k)}=\sum_{m} p_{l}^{m(k+1)}-$ $p_{l}^{(k)}$, thus:

$$
\delta_{l} \leq \bar{p}_{l}-p_{l}^{(k)}
$$

The line flow is brought within its limits.

To summarize, at each iteration $k$ of the algorithm, each MO should solve the following problem to clear its market:

$$
\begin{gathered}
\min _{\mathbf{g}^{m(k)}} \mathbf{c}^{m T} \mathbf{g}^{m(k)} \\
\text { s. t. } \quad \sum_{i} g_{i}^{m(k)}=\sum_{j} d_{j}^{m} \\
0 \leq g_{i}^{m(k)} \leq \bar{g}_{i}^{m} \\
\mathbf{S}_{L}\left(\mathbf{g}^{m(k)}-\mathbf{g}^{m(k-1)}\right) \leq \Delta \mathbf{p}_{L}^{m}
\end{gathered}
$$

where $\mathbf{S}_{L}$ the sub-matrix of $\mathbf{S}$ containing only the rows corresponding to the congested transmission lines and the vector $\Delta \mathbf{p}_{L}^{m}$ is constructed according to (15).

\section{Application to THE Simultaneous CLEARING OF THE OVERLAPPING MARKETS}

\section{A. Test System}

The iterative algorithm has been tested on a small 15bus system, divided into 3 areas-markets, each of which is supposed to be cleared by a different MO (see Fig. 1). It has been made up by triplicating a 5-bus system, connecting the different areas by tie-lines and adjusting the costs-bids of

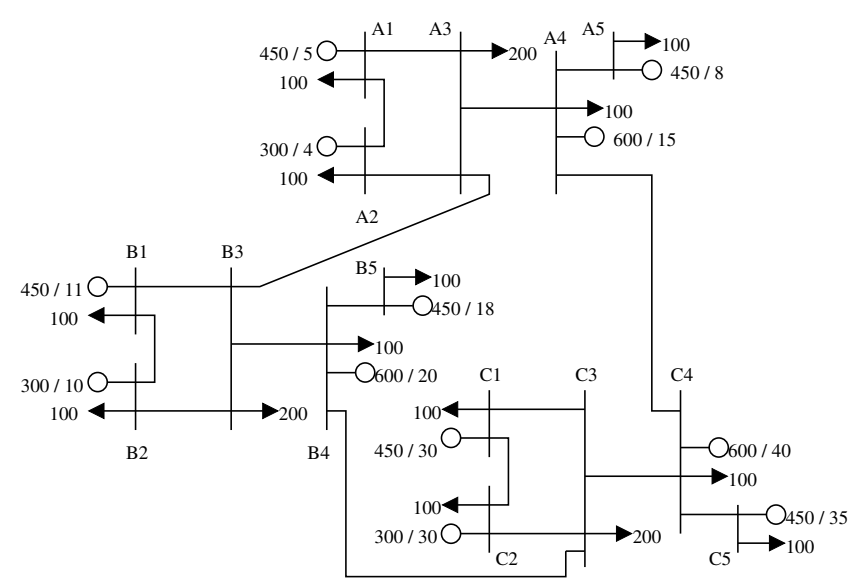

Fig. 1. 3 area test system 
TABLE I

1ST ITERATION; GENERATION CHOSEN BY EACH MO

\begin{tabular}{|c||c|c|c|c|}
\hline Gen & MO-A & MO-B & MO-C & Total \\
\hline \hline gA1 & 150 & 150 & 150 & 450 \\
\hline gA2 & 100 & 100 & 100 & 300 \\
\hline gA4 & 0 & 0 & 0 & 0 \\
\hline gA5 & 150 & 150 & 150 & 450 \\
\hline gB1 & 100 & 100 & 100 & 300 \\
\hline gB2 & 100 & 100 & 100 & 300 \\
\hline gB4 & 0 & 0 & 0 & 0 \\
\hline gB5 & 0 & 0 & 0 & 0 \\
\hline gC5 & 0 & 0 & 0 & 0 \\
\hline
\end{tabular}

TABLE II

1ST ITERATION; RESULTING FLOWS

\begin{tabular}{|c||c|c|c|c|c|c|c|c|}
\hline Line & $p_{l}^{A}$ & $p_{l}^{B}$ & $p_{l}^{C}$ & $p_{l}$ & $\bar{p}_{l}$ & $\Delta p_{l}^{A}$ & $\Delta p_{l}^{B}$ & $\Delta p_{l}^{C}$ \\
\hline \hline A1A2 & 17 & 18 & 18 & 53 & 100 & - & - & - \\
\hline A1A3 & 32 & 133 & 133 & $\mathbf{2 9 8}$ & 150 & -16 & -66 & -66 \\
\hline A2A3 & 17 & 118 & 118 & $\mathbf{2 5 3}$ & 150 & -7 & -48 & -48 \\
\hline A3A4 & 8 & -25 & 175 & 158 & 400 & - & - & - \\
\hline A4A5 & -50 & -150 & -150 & -350 & 400 & - & - & - \\
\hline B1B2 & 0 & 0 & 0 & 0 & 100 & - & - & - \\
\hline B1B3 & 100 & 0 & 100 & $\mathbf{2 0 0}$ & 150 & -25 & 0 & -25 \\
\hline B2B3 & 100 & 0 & 100 & $\mathbf{2 0 0}$ & 150 & -25 & 0 & -25 \\
\hline B3B4 & 41 & 75 & 275 & 391 & 400 & - & - & - \\
\hline B4B5 & 0 & 100 & 0 & 100 & 400 & - & - & - \\
\hline C1C2 & 0 & 0 & 0 & 0 & 100 & - & - & - \\
\hline C1C3 & 0 & 0 & -100 & -100 & 150 & - & - & - \\
\hline C2C3 & 0 & 0 & -100 & -100 & 150 & - & - & - \\
\hline C3C4 & 41 & -125 & -125 & -209 & 400 & - & - & - \\
\hline C4C5 & 0 & 0 & 100 & 100 & 400 & - & - & - \\
\hline A3B3 & -159 & 275 & 75 & 192 & 200 & - & - & - \\
\hline A4C4 & -41 & 125 & 325 & $\mathbf{4 0 9}$ & 200 & - & -58 & -151 \\
\hline B4C3 & 41 & -125 & 275 & 191 & 200 & - & - & - \\
\hline
\end{tabular}

the generators, in order to create areas with cheap and others with more expensive generation. All areas have thus the same amount of load to serve (600 MW each).

On the diagram (Fig. 1), each area is denoted by a different letter (A, B and C). Next to each generator, its maximum production capacity (in MW) as well as its bid (in euros/MWh) are shown. In order to make the evolution of the algorithm easier to follow, each generator capacity has been divided by three, i.e. each generator bids one third of its capacity to every MO. For the same objective of clarity, the same bid per generator has been placed to all the MOs. Generally, it is the choice of each generator how much of its capacity will offer to every market and at what price (the same generator will, in general, bid differently to different markets).

\section{B. Insight into the steps of the algorithm}

It took 9 iterations for the algorithm to converge to a solution. In Tables I - IV the results after the first two iterations are presented. Some comments on the operation of the algorithm are made referring to these results.

In Tables II and IV, the flows that result from the implementation of the generation schedules are presented. For every line, the decomposition of its total flow into three parts is shown, corresponding to the three MOs. Of course, the three parts sum together to the total flow. The lines found overloaded are pointed out using bold numbers. Also, in the last three columns of the tables, the amount of change that each MO
TABLE III

2ND ITERATION; GENERATION CHOSEN BY EACH MO

\begin{tabular}{|c||c|c|c|c|}
\hline Gen & MO-A & MO-B & MO-C & Total \\
\hline \hline gA1 & 125 & 63 & 0 & 188 \\
\hline gA2 & 100 & 73 & 100 & 273 \\
\hline gA4 & 75 & 0 & 0 & 75 \\
\hline gA5 & 150 & 110 & 0 & 260 \\
\hline gB1 & 75 & 100 & 75 & 250 \\
\hline gB2 & 75 & 100 & 75 & 250 \\
\hline gB4 & 0 & 4 & 149 & 153 \\
\hline gB5 & 0 & 150 & 150 & 300 \\
\hline gC5 & 0 & 0 & 51 & 51 \\
\hline
\end{tabular}

TABLE IV

2ND ITERATION; RESULTING FLOWS

\begin{tabular}{|c||c|c|c|c|c|c|c|c|}
\hline Line & $p_{l}^{A}$ & $p_{l}^{B}$ & $p_{l}^{C}$ & $p_{l}$ & $\bar{p}_{l}$ & $\Delta p_{l}^{A}$ & $\Delta p_{l}^{B}$ & $\Delta p_{l}^{C}$ \\
\hline \hline A1A2 & 9 & -3 & -35 & -29 & 100 & - & - & - \\
\hline A1A3 & 16 & 67 & 35 & 118 & 150 & 4 & 18 & 10 \\
\hline A2A3 & 9 & 70 & 65 & 144 & 150 & 0 & 3 & 3 \\
\hline A3A4 & -46 & -43 & 175 & 86 & 400 & - & - & - \\
\hline A4A5 & -50 & -110 & 0 & -160 & 400 & - & - & - \\
\hline B1B2 & 0 & 0 & 0 & 0 & 100 & - & - & - \\
\hline B1B3 & 75 & 0 & 75 & 150 & 150 & 0 & 0 & 0 \\
\hline B2B3 & 75 & 0 & 75 & 150 & 150 & 0 & 0 & 0 \\
\hline B3B4 & 21 & -21 & 75 & 75 & 400 & - & - & - \\
\hline B4B5 & 0 & -50 & -150 & -200 & 400 & - & - & - \\
\hline C1C2 & 0 & 0 & 0 & 0 & 100 & - & - & - \\
\hline C1C3 & 0 & 0 & -100 & -100 & 150 & - & - & - \\
\hline C2C3 & 0 & 0 & -100 & -100 & 150 & - & - & - \\
\hline C3C4 & 21 & -67 & -26 & -72 & 400 & - & - & - \\
\hline C4C5 & 0 & 0 & 49 & 49 & 400 & - & - & - \\
\hline A3B3 & -129 & 179 & -75 & -25 & 200 & - & - & - \\
\hline A4C4 & -21 & 67 & 175 & $\mathbf{2 2 1}$ & 200 & - & -6 & -15 \\
\hline B4C3 & 21 & -67 & 374 & $\mathbf{3 2 8}$ & 200 & -7 & - & -121 \\
\hline
\end{tabular}

must at least enforce on the line flow at the next iteration is presented. A dash (-) in this field means that the MO has no obligation regarding the corresponding line flow when clearing its market at the next iteration.

Once a line gets overloaded a constraint is imposed on its flow, according to (17d). An inequality constraint remains included in all the subsequent iterations for that line, even if the overload is relieved. The reason for doing so is to avoid that the line gets overloaded again in a future iteration. Therefore, at each iteration, the constraint is updated according to the new flow values.

The reader can ascertain that, for each line $l$ that has been overloaded at least once, adding together the three $\Delta p_{l}^{m}$ corresponding to the three MOs gives a total $\Delta p_{l}$ which is exactly equal to the difference between the present flow and the maximum one: $p_{l}+\Delta p_{l}=\bar{p}_{l}$. This holds true irrespective of whether the line is presently overloaded or not. If the line is overloaded, the constraint distributes among the MOs the effort to bring back the line flow within the feasible limits, while if it is not overloaded, the constraint shares the remaining line capacity among the MOs. In both cases the used rule keeps the proportionality with the responsibility that each $\mathrm{MO}$ has on the flow. For instance, even if the lines A1A3 and A2A3 are no longer overloaded at the 2nd iteration (see Table IV), the corresponding constraint $(17 \mathrm{~d})$ remains in the optimization problem, as can be verified from the positive values of the three $\Delta p_{l}^{m}$ in Table IV: the MOs can now use the remaining 
TABLE V

FINAL POINT; GENERATION CHOSEN BY EACH MO

\begin{tabular}{|c|c||c|c|c|c|}
\hline Gen & bid & MO-A & MO-B & MO-C & Total \\
\hline \hline gA1 & 5 & 134 & 99 & 17 & 250 \\
\hline gA2 & 4 & 96 & 59 & 95 & 250 \\
\hline gA4 & 15 & 94 & 0 & 0 & 94 \\
\hline gA5 & 8 & 150 & 80 & 0 & 230 \\
\hline gB1 & 11 & 26 & 100 & 124 & 250 \\
\hline gB2 & 10 & 100 & 100 & 50 & 250 \\
\hline gB4 & 20 & 0 & 12 & 0 & 12 \\
\hline gB5 & 18 & 0 & 150 & 114 & 264 \\
\hline gC1 & 30 & 0 & 0 & 28 & 28 \\
\hline gC2 & 30 & 0 & 0 & 100 & 100 \\
\hline gC4 & 40 & 0 & 0 & 0 & 0 \\
\hline gC5 & 35 & 0 & 0 & 72 & 72 \\
\hline
\end{tabular}

available capacity of the line, but to a limited extent so that the line remains within its limits.

Taking a look in Table II, one can see that for the tie-line $\mathrm{A} 4 \mathrm{C} 4, \mathrm{MO}-\mathrm{A}$ is not attributed any constraint at all, even if the line is overloaded. All the effort is shared between MO$\mathrm{B}$ and MO-C (indeed $\Delta p_{l}^{B}+\Delta p_{l}^{C}=\bar{p}_{l}-p_{l}$ ). MO-A is allowed to cause whatever change on the line flow at the next iteration. The reason is that MO-A is creating a counterflow on the line. Indeed, it is deemed more fair and efficient not to apply a constraint to the MOs causing a counterflow on a congested line. The change needed is shared among the other MOs, assuming that the counterflowing ones will continue to counterflow by the same amount. If this turns out not to happen, a new constraint will be applied to the MO at the next iteration of the algorithm.

In this respect, it is interesting to see why line $\mathrm{A} 4 \mathrm{C} 4$ remained overloaded after the 2nd iteration, while the other four overloaded ones (lines A1A3, A2A3, B1B3 and B2B3) all came back within their limits. One can see that the line remained overloaded by an amount $p_{l}^{(2)}-\bar{p}_{l}=21 M W=$ $p_{l}^{A(2)}-p_{l}^{A(1)}$, that is to say by the amount that MO-A decreased its counterflowing contribution. MO-A decreased its contribution because it was obliged to do so in order to satisfy its constraints (relative to lines A1A3, A2A3, B1B3 and B2B3). In fact, when all MOs are assigned responsibility for an overload (no one counterflows), then, at the next step, the line will for sure be unloaded, since (9b) holds true. On the contrary, when at least one MO is counterflowing an overloaded line, then it is possible that the line remains overloaded at the next step. Even a non-overloaded line may become overloaded if there is one (or more) MO that is counterflowing. However, this does not really cause a problem; these calculations are nothing but intermediate steps of a process which converges to the sought operating point. At the end of the procedure no line remains overloaded.

In Tables V and VI the generation schedules and the flows at the final equilibrium point (to be actually implemented) are presented. No line is overloaded at this point. Lines A1A3, $\mathrm{A} 2 \mathrm{~A} 3, \mathrm{~B} 1 \mathrm{~B} 3, \mathrm{~B} 2 \mathrm{~B} 3, \mathrm{~A} 4 \mathrm{C} 4$ and $\mathrm{B} 4 \mathrm{C} 3$ are fully used. It is interesting to mention that these are the lines that, from the first steps of the algorithm, turned out to be the most crucial for the satisfaction of the most economic generation schedules.

It is also noteworthy that MO-A finally manages to allocate
TABLE VI

FINAL POINT; RESULTING FLOWS

\begin{tabular}{|c||c|c|c|c|c|c|c|c|}
\hline Line & $p_{l}^{A}$ & $p_{l}^{B}$ & $p_{l}^{C}$ & $p_{l}$ & $\bar{p}_{l}$ & $\Delta p_{l}^{A}$ & $\Delta p_{l}^{B}$ & $\Delta p_{l}^{C}$ \\
\hline \hline A1A2 & 13 & 14 & -27 & 0 & 100 & - & - & - \\
\hline A1A3 & 21 & 85 & 44 & 150 & 150 & 0 & 0 & 0 \\
\hline A2A3 & 9 & 73 & 68 & 150 & 150 & 0 & 0 & 0 \\
\hline A3A4 & -57 & -21 & 154 & 76 & 400 & - & - & - \\
\hline A4A5 & -50 & -80 & 0 & -130 & 400 & - & - & - \\
\hline B1B2 & -26 & 0 & 26 & 0 & 100 & - & - & - \\
\hline B1B3 & 52 & 0 & 98 & 150 & 150 & 0 & 0 & 0 \\
\hline B2B3 & 74 & 0 & 76 & 150 & 150 & 0 & 0 & 0 \\
\hline B3B4 & 14 & -21 & 131 & 124 & 400 & - & - & - \\
\hline B4B5 & 0 & -50 & -114 & -164 & 400 & - & - & - \\
\hline C1C2 & 0 & 0 & -25 & -25 & 100 & - & - & - \\
\hline C1C3 & 0 & 0 & -47 & -47 & 150 & - & - & - \\
\hline C2C3 & 0 & 0 & -25 & -25 & 150 & - & - & - \\
\hline C3C4 & 14 & -59 & -27 & -72 & 400 & - & - & - \\
\hline C4C5 & 0 & 0 & 28 & 28 & 400 & - & - & - \\
\hline A3B3 & -112 & 178 & -42 & 24 & 200 & - & - & - \\
\hline A4C4 & -14 & 60 & 154 & 200 & 200 & - & 0 & 0 \\
\hline B4C3 & 14 & -60 & 246 & 200 & 200 & 0 & - & 0 \\
\hline
\end{tabular}

mainly the less expensive generators (located geographically in its area), while on the other hand, MO-C is mostly obliged to resort to some expensive generators (geographically located in its own area). This makes sense since MO-C is the main responsible for loading the tie-lines $\mathrm{A} 4 \mathrm{C} 4$ and $\mathrm{B} 4 \mathrm{C} 3$, and, consequently, it is the one who is mainly assigned the effort for unloading.

\section{Evaluation of the results}

Table VII presents the generation schedules that result from a global market clearing for the entire interconnection. The following optimization problem was solved, including all areas (index $m$ ) and buses (index $i$ ):

$$
\begin{array}{ll} 
& \min _{\mathbf{g}^{m}} \sum_{m} \mathbf{c}^{m T} \mathbf{g}^{m} \\
\text { s. t. } & \sum_{i} g_{i}^{m}=\sum_{j} d_{j}^{m} \\
& 0 \leq g_{i}^{m} \leq \bar{g}_{i}^{m} \\
-\overline{\mathbf{p}} \leq \mathbf{S}(\mathbf{g}-\mathbf{d}) \leq \overline{\mathbf{p}}
\end{array}
$$

The vectors $\mathbf{g}$ and $\mathbf{d}$ are given by Eq. (3) and (4). It should be noted that (18b) contains three equality constraints, each one preserving the power balance of an MO $m$. Thus, the schedules presented in Table VII are globally optimal, given the generator decisions of where to bid.

In Table VIII the resulting costs are shown. For the $m$-th MO they have been computed according to:

$$
C^{m}=\sum_{i} c_{i}^{m} g_{i}^{m}
$$

with $i$ corresponding to all the generators that are producing for the MO $m$.

Three individual market clearings, without cross region bidding, in which each MO considers only the generators located in its area were also performed for comparison. To do so, three optimization problems were solved, one for each area, each of them referring only to variables and constraints geographically 
TABLE VII

GLOBAL MARKET CLEARING

\begin{tabular}{|c|c||c|c|c|c|}
\hline Gen & bid & MO-A & MO-B & MO-C & Total \\
\hline \hline gA1 & 5 & 150 & 50 & 50 & 250 \\
\hline gA2 & 4 & 100 & 100 & 50 & 250 \\
\hline gA4 & 15 & 0 & 0 & 0 & 0 \\
\hline gA5 & 8 & 150 & 150 & 0 & 300 \\
\hline gB1 & 11 & 0 & 100 & 150 & 250 \\
\hline gB2 & 10 & 50 & 100 & 100 & 250 \\
\hline gB4 & 20 & 0 & 0 & 0 & 0 \\
\hline gB5 & 18 & 150 & 0 & 150 & 300 \\
\hline gC1 & 30 & 0 & 0 & 0 & 0 \\
\hline gC2 & 30 & 0 & 100 & 100 & 200 \\
\hline gC4 & 40 & 0 & 0 & 0 & 0 \\
\hline gC5 & 35 & 0 & 0 & 0 & 0 \\
\hline
\end{tabular}

located within the area. As a result, the generators of each area produce all together exactly the amount of the area's total load. By chance no line got overloaded. However this could happen in general, since no area considers the effect of its schedule on the other areas.

TABLE VIII COSTS COMPARISON

\begin{tabular}{|c||c|c|c|c|}
\hline Cost: & MO-A & MO-B & MO-C & Total \\
\hline \hline global clearing & 5550 & 6950 & 8800 & 21300 \\
\hline proposed iter. algorithm & 4950 & 6412 & 10740 & 22102 \\
\hline independent clearing & 3050 & 7050 & 18500 & 28600 \\
\hline
\end{tabular}

The proposed iterative algorithm is not equivalent to solving the global problem (18) and, thus, the resulting schedules are not globally optimal (see last column of Table VIII).

In fact, the proposed algorithm allows cross market bidding while, first, respecting the individuality of each market (no global market clearing is attempted) and, second, managing the resulting congestion according to a specific policy (as described in Section III). This policy is highlighted by comparing the individual MOs costs resulting from the iterative algorithm and the global clearing (first two rows in Table VIII, columns 2-4); in the global clearing the total cost has been minimized by obtaining a significantly lower cost for MO-C even if the costs of MOs A and B were higher than in the iterative algorithm. This difference reflects the fact that MO-C, during the execution of the iterative algorithm, has to deallocate generation from areas A and B in order to alleviate the congestion of the tie-lines for which it is mainly responsible.

Nonetheless, the total social cost resulting from the iterative algorithm (22102 euros) is just $3.76 \%$ higher than the minimum social cost (21300 euros) that can be obtained and significantly lower $(29.4 \%)$ than the social cost resulting from the independent market clearings (28600 euros). This shows that the congestion management policy used in this paper goes with the objective of dispatching as much as possible the cheapest generators, while in the same time preserving the independency of the different markets.

\section{CONCLUSION}

The possibility of allowing external actors to bid in whatever market of an interconnection has been investigated in this paper. The situation has been modeled as the existence of several overlapping markets. An iterative algorithm that permits a simultaneous clearing of all markets and leads to feasible schedules has been proposed. The algorithm has been illustrated on a test system allowing to highlight some of its attractive features.

Based on the credible (at least in Europe) assumptions of sharing a common model of the interconnection and making use of a "neutral" coordinating entity (performing, practically, just some central computations), the presented algorithm is easy to implement and beneficial for the goal of moving towards a common electricity market from the participants perspective. As to the first, only a small set of linear constraints are communicated to the MOs and can be easily incorporated into their market clearing procedures. Regarding the second, market participants can freely choose the MO of their preference; this can be based on a combination of criteria, like expected prices, different financial or IT tools, favoring of clean energies and other.

Within this paper, a small test system has been chosen as an example to draw the reader's attention on the features of the algorithm. However, the algorithm should easily scale up to large systems; only a few lines are expected to tend to get congested. Preliminary tests with the 3-area IEEE RTS-196, to be reported shortly, have also resulted in fast convergence (less than 10 iterations in all cases).

In the presented algorithm, every generator decides a priori how much of its available capacity will offer to each market. This may result in generation capacity not been finally allocated by any MO even if it would be useful to some of them. An interesting extension will be to allow shifting generation capacity from one market to another during the execution of the algorithm.

\section{REFERENCES}

[1] "Development and Implementation of a Coordinated Model for regional and Inter-regional Congestion Management", ETSO-EuroPex Interim Report, April 2008, http://www.etso-net.org

[2] O. Gjerde, J. Bogas, A. DiCaprio, O.B. Fosso, S. Cisneiros, M. Uusitalo, "Congestion management: The system operators challenge to balance transmission transfer capacity with an acceptable security level", CIGRE/IEEE PES International Symposium, pp. 120-127, 2005.

[3] BELPEX, APX, Powernext, "Trilateral Market Coupling: Algorithm Appendix", March 2006, available online: http://www.belpex.be/uploads/media/Algorithm Appendix v4.6 PPOadaptations final.pdf

[4] "Implementation Study: A report for the MoU signatories on the design of the market coupling solution in the Central West European (CWE) region, by the CWE MC Project", August 2008, available online: http://www.apxgroup.com/uploads/media/Implementation Study with Logo ta.pdf

[5] L. Vandezande, L. Meeus, R. Belmans, "The next step in the Central Western European electricity market: cross-border balancing", Bourses d'énergie, no. 1, pp. 19-24, March 2008.

[6] Shangyou Hao, "Decentralized Approach to Intermarket Congestion Management", IEEE Transactions on Power Systems, vol. 20, no. 2, pp. 675683, May 2005.

[7] R.D. Christie, B.F. Wollenberg, I. Wangensteen, "Transmission Management in the Deregulated Environment", Proceedings of the IEEE, vol. 88, is. 2, pp. $170-195$, Feb. 2000.

[8] Kai Liu, Yixin Ni, Felix F. Wu, T.S. Bi, "Decentralized Congestion Management for Multilateral Transactions Based on Optimal Resource Allocation", IEEE Transactions on Power Systems, vol. 22, no. 4, pp. 1835-1842, November 2007. 